biopsy. Early brain biopsy is recommended in cases of obscure encephalitis in afebrile immunocompromised patients who present with focal status epilepticus.

\title{
NEUROLOGIC COMPLICATIONS OF INFLUENZA A INFECTION
}

Eight children aged 5 months to 9 years with neurologic complications associated with influenza A were identified by a review of records of patients admitted to Texas Children's Hospital, Houston, during an early and severe outbreak in October and November 2003. None had received the influenza vaccine. Presenting symptoms included seizures in 4 patients, altered mental status in 3, and mutism in 1; all occurred within 3 weeks of an upper respiratory infection onset, and all had symptoms and signs of influenza. The seizure manifestations were not typical of febrile seizures; 3 patients had laboratory or imaging evidence of direct CNS involvement, one was 9 years of age, and 1 had several afebrile seizures 3 days after the first seizure. Influenza A viral antigen (H3N2 A/Fujian/411/2002like) was detected in nasal wash samples of 7 patients, and the virus was isolated in cultures of 6 nasal wash specimens. The virus was also isolated from the CSF of 1 of these patients. None had serum metabolic abnormalities or other CSF abnormalities. Brain imaging was abnormal in 3 patients. Antiviral treatment was used in 5. All 8 recovered, 6 completely and 2 with sequelae. (Maricich SM, Neul JL, Lotze TE, et al. Neurologic complications associated with influenza A in children during the 2003-2004 influenza season in Houston, Texas. Pediatrics November 2004;114:e626-e633). (Reprints: Stephen M Maricich PhD MD, Fellow in Child Neurology, CCC 17.10, 6621 Fannin St, Houston, TX 77030).

COMMENT. During the 2003-2004 influenza season in the United States, 142 deaths occurred in children infected (CDC MMWR 2004;52:1286-1288). The influenza A (H3N2) Fujian strain identified in the Houston outbreak was found in the majority of encephalopathy cases reported in Japan, and this strain may have an affinity for the CNS (Morishima T, et al. Clin Infect Dis 2002;35:512-517). Influenza A infection is an important cause of febrile seizures, especially in Japan (Kawada J-I, et al. J Infect Dis 2003;188:690698) and China (Chiu SS, et al. Pediatrics 2001;108:e-63), but the association is less frequent in the United States, where herpesvirus (HHV)-6 accounts for one-third of all first time febrile seizures in children $<2$ years old (Hall CB, et al. N Engl J Med 1994;331:432438). Seizures were the most common presenting neurologic complication of influenza A infection in the Houston outbreak. In this series, the seizure manifestations were not typical of febrile seizures and were classified as encephalopathic. The distinction between encephalopathy with seizures and complex febrile seizures is often difficult, but systemic cytokine responses may be involved in both (Kawada J-I, et al, 2003: Millichap JG, Millichap JJ. Influenza virus and febrile convulsions. J Infect Dis 2004;189:564). Studies of the role of viral infections in the cause of febrile convulsions is a relatively neglected field of research in pediatric neurology, and further work is needed to elucidate the mechanism of the seizure and the differentiation of simple and complex febrile convulsions. More severe neurologic complications of influenza A infection have included acute disseminated encephalomyelitis, transverse myelitis, Guillain-Barre syndrome, frontal lobe syndromes, and acute necrotizing encephalopathy (Studahl M. J Clin Virol 2003;28:225-232). 